\title{
Symmetry approach and the generalized Korteweg-de Vries equation with variable coefficients
}

Wescley L. Souza* and Érica M. Silva ${ }^{\dagger}$

Instituto de Física - UFMT, Brazil

E-mail: wescley@fisica.ufmt.br, erica@fisica.ufmt.br

Using Lie symmetry arguments, we consider a class of nonlinear KdV-type equations which are usually denoted by $K(m, n)$. We start with the 4-dimensional Lie algebra of the ordinary KdV equation to derive and classify $K(m, n)$ equations with space- and time-dependent coefficients.

5th International School on Field Theory and Gravitation,

April 20 - 242009

Cuiabá city, Brazil

${ }^{*}$ Poster Section

† Corresponding author 


\section{Introduction}

More than one decade ago, the celebrated Korteweg-de Vries (KdV) equation,

$$
u_{t}+\left[u^{2}+u_{x x}\right]_{x}=0,
$$

was generalized to a class of nonlinear equations, named as $K(m, n)$ equations [1], given by

$$
u_{t}+\left[u^{m}+\left(u^{n}\right)_{x x}\right]_{x}=0, m>0,1<n \leq 3 .
$$

For some values of $m$ and $n$, solutions of $K(m, n)$ equations have compact support and independent wave amplitude width [1]. This kind of solution is called compacton and, in nature, is different from a KdV soliton, that narrows as the amplitude increases.

In the classical soliton theory, integrability and elastic collisions are closely connected but, in the realm of the $K(m, n)$ equations, albeit some conservation laws have been derived, it is not known whether these equations are integrable [2]. A lot of effort has been carried out in order to understand the nonlinear mechanism that underlies processes described by $K(m, n)$ equations [ [3, 4 , 5], including an analogous generalization of the Sine-Gordon equation [6]. Lie symmetry methods have also been used for this purpose, and a partial symmetry classification of $K(m, n)$ equations has been achieved [7, 8, 9,

In order to derive generalizations of a partial differential equation, a standard procedure is to choose a starting symmetry, that usually is considered as the symmetry of a more restrictive set of equations. This approach has been adopted to obtain, for instance, generalized Fokker-Planck equations which admits the Lie symmetry of a specific diffusion equation [10]. Our goal here is to follow along this line and study and classify some $K(m, n)$ equations. Considering the Lie symmetry algebra of the classical $\mathrm{KdV}$ equation, say $\ell_{K d V}$, then we proceed to find all equations in a given class of $K(m, n)$ equations that are invariant under $\ell_{K d V}$. The class we have studied is the nonlinear $K(m, n)$ equations with space- and time-dependent coefficients.

\section{Determining equations}

Let us start by noting that, associated with Eq. (1.1), there is a set of generators of Lie symmetries given by

$$
\begin{aligned}
& X_{1}=\partial_{x}, \\
& X_{2}=\partial_{t}, \\
& X_{3}=2 t \partial_{x}+\partial_{u}, \\
& X_{4}=x \partial_{x}+3 t \partial_{t}-2 u \partial_{u} .
\end{aligned}
$$

These generators fulfill the following commutation relations

$$
\begin{aligned}
& {\left[X_{1}, X_{4}\right]=X_{1},} \\
& {\left[X_{2}, X_{3}\right]=2 X_{1},} \\
& {\left[X_{2}, X_{4}\right]=3 X_{2},} \\
& {\left[X_{3}, X_{4}\right]=-2 X_{3},} \\
& {\left[X_{1}, X_{2}\right]=\left[X_{1}, X_{3}\right]=0 .}
\end{aligned}
$$


We use this $\mathrm{KdV}$ equation Lie algebra, denoted here by $\ell_{K d V}$, to derive $K(m, n)$ equations. Let us consider a nonlinear generalization of Eq. (1.2) with space- and time-dependent coefficients, that is,

$$
u_{t}+\left[f u^{m}+g\left(u^{n}\right)_{x x}\right]_{x}=0,
$$

where $f=f(x, t)$ and $g=g(x, t)$. This equation is written as

$$
u_{t}+a_{0} u^{m}+a_{1} u^{m-1} u_{x}+a_{2} u^{n-2} u_{x}^{2}+a_{3} u^{n-1} u_{x x}+a_{4} u^{n-3} u_{x}^{3}+a_{5} u^{n-2} u_{x} u_{x x}+a_{6} u^{n-1} u_{x x x}=0,
$$

where

$$
\begin{aligned}
& a_{0}=f_{x}, \\
& a_{1}=m f, \\
& a_{2}=n(n-1) g_{x}, \\
& a_{3}=n g_{x}, \\
& a_{4}=n(n-1)(n-2) g, \\
& a_{5}=3 n(n-1) g, \\
& a_{6}=n g .
\end{aligned}
$$

A vector field of the form

$$
X=\eta(u, x, t) \partial_{u}+\theta_{1}(u, x, t) \partial_{x}+\theta_{2}(u, x, t) \partial_{t}
$$

is a symmetry generator of Eq. (1.1) if Eq. (2.3) is form invariant under the infinitesimal transformation $x^{\prime}=x+\varepsilon \theta_{1}, t^{\prime}=t+\varepsilon \theta_{2}$, and $u^{\prime}=u+\varepsilon \eta$. This leads to the following set of determining equations:

$$
\begin{aligned}
& e q_{1}:\left(a_{6} u^{n-1} \partial_{u u u}+a_{5} u^{n-2} \partial_{u u}+2 a_{4} u^{n-3} \partial_{u}\right) \theta_{2}(u, x, t)=0 \\
& e q_{2}:\left(6 a_{6} u^{n-1} \partial_{u x}+2 a_{5} u^{n-2} \partial_{x}+2 a_{3} u^{n-1} \partial_{u}\right) \theta_{2}(u, x, t)=0 \\
& e q_{3}:\left(a_{6} u^{n-1} \partial_{u u u}+a_{5} u^{n-2} \partial_{u u}-a_{4} u^{n-3} \partial_{u}\right) \theta_{1}(u, x, t)=0 \\
& e q_{4}:\left(3 a_{6} u^{n-1} \partial_{u}\right) \theta_{1}(u, x, t)=0 \\
& e q_{5}:\left(3 a_{6} u^{n-1} \partial_{u u}+a_{5} u^{n-2} \partial_{u}\right) \theta_{2}(u, x, t)=0 \\
& e q_{6}:\left(3 a_{6} u^{n-1} \partial_{x x}+2 a_{3} u^{n-1} \partial_{x}\right) \theta_{2}(u, x, t)=0 \\
& e q_{7}:\left(3 a_{6} u^{n-1} \partial_{u x}+a_{5} u^{n-2} \partial_{x}\right) \theta_{2}(u, x, t)=0 \\
& e q_{8}:\left(3 a_{6} u^{n-1} \partial_{u u}+2 a_{5} u^{n-2} \partial_{u}\right) \theta_{2}(u, x, t)=0 \\
& e q_{9}:\left(3 a_{6} u^{n-1} \partial_{u}\right) \theta_{2}(u, x, t)=0 \\
& e q_{10}:\left(6 a_{6} u^{n-1} \partial_{u u}\right) \theta_{1}(u, x, t)=0 \\
& e q_{11}: 3 a_{6} u^{n-1} \partial_{x} \theta_{2}(u, x, t)=0 \\
& e q_{12}:\left(3 \partial_{u}\right) \theta_{1}(u, x, t)-\left(3 a_{6} u^{n-1} \partial_{u x x}+a_{5} u^{n-2} \partial_{x x}+2 a_{3} u^{n-1} \partial_{u x}+2 a_{2} u^{n-2} \partial_{x}\right) \theta_{2}(u, x, t)=0, \\
& e q_{13}:\left(3 a_{6} u^{n-1} \partial_{u u x}+2 a_{5} u^{n-2} \partial_{u x}+3 a_{4} u^{n-3} \partial_{x}+a_{3} u^{n-1} \partial_{u u}+a_{2} u^{n-2} \partial_{u}\right) \theta_{2}(u, x, t)=0
\end{aligned}
$$




$$
\begin{aligned}
e q_{14}:( & \left.n u^{-1}-u\right) \eta(u, x, t)+\left(\frac{\left(\partial_{x} a_{6}\right)}{a_{6}}-3 \partial_{x}\right) \theta_{1}(u, x, t) \\
& +\left(a_{6} u^{n-1} \partial_{x x x}+\frac{\left(\partial_{t} a_{6}\right)}{a_{6}}+a_{3} u^{n-1} \partial_{x x}+a_{1} u^{m-1} \partial_{x}-\partial_{u}+\partial_{t}\right) \theta_{2}(u, x, t)=0, \\
e q_{15}:( & \left.3 a_{6} u^{n-1} \partial_{u x x}+a_{5} u^{n-2} \partial_{x x}+2 a_{3} u^{n-1} \partial_{u x}+a_{1} u^{m-2} m\right) \eta(u, x, t) \\
& -\left(a_{6} u^{n-1} \partial_{x x x}+a_{3} u^{n-1} \partial_{x x}+a_{1} \frac{\left(\partial_{x} a_{6}\right)}{a_{6}} u^{m-1}-4 a_{0} u^{m} \partial_{u}+\partial_{t}\right) \theta_{1}(u, x, t) \\
& -\left(a_{1}\left(\frac{\left(\partial_{t} a_{6}\right)}{a_{6}}-\frac{\left(\partial_{t} a_{1}\right)}{a_{1}}\right) u^{m-1}\right) \theta_{2}(u, x, t)=0, \\
e q_{16}:( & \left.3 a_{6} u^{n-1} \partial_{u u x}+2 a_{5} u^{n-2} \partial_{u x}+3 a_{4} u^{n-3} \partial_{x}-a_{3} u^{n-1} \partial_{u u}+a_{2}\left(u^{n-2} \partial_{u}-u^{n-3}\right)\right) \eta(u, x, t) \\
& -\left(3 a_{6} u^{n-1} \partial_{u x x}+a_{5} u^{n-2} \partial_{x x}+2 a_{3} u^{n-1} \partial_{u x}+a_{2} u^{n-2}\left(\frac{\left(\partial_{x} a_{6}\right)}{a_{6}}-\partial_{x}\right)\right) \theta_{1}(u, x, t) \\
& -\left(\left(\partial_{x} a_{2}\right) u^{n-2}-3 a_{1} u^{m-1} \partial_{u}\right) \theta_{1}(u, x, t)-\left(a_{2}\left(\frac{\left(\partial_{t} a_{6}\right)}{a_{6}}-\frac{\left(\partial_{t} a_{2}\right)}{a_{2}}\right) u^{n-2}\right) \theta_{2}(u, x, t)=0, \\
e q_{17}:( & \left.a_{6} u^{n-1} \partial_{u u u}+a_{5} u^{n-2} \partial_{u u}+2 a_{4}\left(u^{n-3} \partial_{u}-u^{n-4}\right)\right) \eta(u, x, t) \\
& -\left(3 a_{6} u^{n-1} \partial_{u u x}+2 a_{5} u^{n-2} \partial_{u x}+a_{4} \frac{\left(\partial_{x} a_{6}\right)}{a_{6}} u^{n-3}-\left(\partial_{x} a_{4}\right) u^{n-3}+a_{3} u^{n-1} \partial_{u u}\right) \theta_{1}(u, x, t) \\
& -\left(2 a_{2} u^{n-2} \partial_{u}\right) \theta_{1}(u, x, t)-\left(a_{4}\left(\frac{\left(\partial_{t} a_{6}\right)}{a_{6}}-\frac{\left(\partial_{t} a_{4}\right)}{a_{4}}\right) u^{n-3}\right) \theta_{2}(u, x, t)=0,
\end{aligned}
$$

$e q_{18}:\left(3 a_{6} u^{n-1} \partial_{u u}+a_{5}\left(u^{n-2} \partial_{u}-u^{n-3}\right)\right) \eta(u, x, t)$

$$
\begin{aligned}
& -\left(9 a_{6} u^{n-1} \partial_{u x}+a_{5} \frac{\left(\partial_{x} a_{6}\right)}{a_{6}} u^{n-2}-\left(\partial_{x} a_{5}\right) u^{n-2}-a_{3} u^{n-1} \partial_{u}\right) \theta_{1}(u, x, t) \\
& -\left(a_{5}\left(\frac{\left(\partial_{t} a_{6}\right)}{a_{6}}-\frac{\left(\partial_{t} a_{5}\right)}{a_{5}}\right) u^{n-2}\right) \theta_{2}(u, x, t)=0,
\end{aligned}
$$

$e q_{19}:\left(a_{6} u^{n-1} \partial_{x x x}+a_{3} u^{n-1} \partial_{x x}+a_{1} u^{m-1} \partial_{x}+a_{0}\left(u^{m} \partial_{u}+u^{m-1}(m-n+1)\right)+\partial_{t}\right) \eta(u, x, t)$

$$
\begin{aligned}
& -\left(a_{0} u^{m}\left(\frac{\left(\partial_{x} a_{6}\right)}{a_{6}}-\frac{\left(\partial_{x} a_{0}\right)}{a_{0}}-3 \partial_{x}\right)\right) \theta_{1}(u, x, t) \\
& -\left(a_{0}\left(\frac{\left(\partial_{t} a_{6}\right)}{a_{6}}-\frac{\left(\partial_{t} a_{0}\right)}{a_{0}}\right) u^{m}\right) \theta_{2}(u, x, t)=0,
\end{aligned}
$$

$e q_{20}:\left(3 a_{6} u^{n-1} \partial_{u x}+a_{5} u^{n-2} \partial_{x}\right) \eta(u, x, t)$

$$
\begin{aligned}
& -\left(3 a_{6} u^{n-1} \partial_{x x}+a_{3} u^{n-1}\left(\frac{\left(\partial_{x} a_{6}\right)}{a_{6}}-\frac{\left(\partial_{x} a_{3}\right)}{a_{3}}-\partial_{x}\right)\right) \theta_{1}(u, x, t) \\
& -\left(a_{5}\left(\frac{\left(\partial_{t} a_{6}\right)}{a_{6}}-\frac{\left(\partial_{t} a_{3}\right)}{a_{3}}\right) u^{n-1}\right) \theta_{2}(u, x, t)=0 .
\end{aligned}
$$

The substitution of the dominant derivative from Eq. (1.1) into the determining system of infinitesimal symmetry transformations results in a set of equations in the coefficients $a_{i}, i=0, \ldots, 6$.

\section{The $K(m, n)$ equations with variable coefficients}

In order to find the $K(m, n)$ generalized equations, we impose that Eq. (2.3) admits subalgebras of $\ell_{K d V}$ as symmetry Lie algebras. Recalling that $\ell_{K d V}$ is spanned by the generators $X_{i}, i=1,2,3,4$, 
we have the following cases.

Symmetry $\left\{\mathbf{X}_{4}\right\}$ : By imposing this symmetry generator to Eq. (2.3), the resulting system of equations in the coefficients $a_{i}, i=0, \ldots, 6$, is

$$
\begin{aligned}
& e q_{1}: \frac{1}{2} u^{m}\left(a_{0}\left(\frac{\left(\partial_{x} a_{6}\right)}{a_{6}} x+3 \frac{\left(\partial_{t} a_{6}\right)}{a_{6}} t+2\left(m-n-\frac{3}{2}\right)\right)-\left(\partial_{x} a_{0}\right) x-3\left(\partial_{t} a_{0}\right) t\right)=0, \\
& e q_{2}: \frac{1}{2} u^{m-1}\left(a_{1}\left(\frac{\left(\partial_{x} a_{6}\right)}{a_{6}} x+3 \frac{\left(\partial_{t} a_{6}\right)}{a_{6}} t+2(m-n-1)\right)-\left(\partial_{x} a_{1}\right) x-3\left(\partial_{t} a_{1}\right) t\right)=0, \\
& e q_{3}: \frac{1}{2} u^{n-2}\left(a_{2}\left(\frac{\left(\partial_{x} a_{6}\right)}{a_{6}} x+3 \frac{\left(\partial_{t} a_{6}\right)}{a_{6}} t-1\right)-\left(\partial_{x} a_{2}\right) x-3\left(\partial_{t} a_{2}\right) t\right)=0 \\
& e q_{4}: \frac{1}{2} u^{n-1}\left(a_{3}\left(\frac{\left(\partial_{x} a_{6}\right)}{a_{6}} x+3 \frac{\left(\partial_{t} a_{6}\right)}{a_{6}} t-1\right)-\left(\partial_{x} a_{3}\right) x-3\left(\partial_{t} a_{3}\right) t\right)=0 \\
& e q_{5}: \frac{1}{2} u^{n-3}\left(a_{4}\left(\frac{\left(\partial_{x} a_{6}\right)}{a_{6}} x+3 \frac{\left(\partial_{t} a_{6}\right)}{a_{6}} t\right)-\left(\partial_{x} a_{4}\right) x-3\left(\partial_{t} a_{4}\right) t\right)=0 \\
& e q_{6}: \frac{1}{2} u^{n-2}\left(a_{5}\left(\frac{\left(\partial_{x} a_{6}\right)}{a_{6}} x+3 \frac{\left(\partial_{t} a_{6}\right)}{a_{6}} t\right)-\left(\partial_{x} a_{5}\right) x-3\left(\partial_{t} a_{5}\right) t\right)=0 \\
& e q_{7}: 2\left(\frac{\left(\partial_{x} a_{6}\right)}{a_{6}} x+3 \frac{\left(\partial_{t} a_{6}\right)}{a_{6}} t\right)-n+1=0 .
\end{aligned}
$$

Taking the general solution of the system given by Eqs. (3.1) into Eqs. (2.4) implies that

$$
u_{t}+\left[f u^{m}+g\left(u^{n}\right)_{x x}\right]_{x}=0,
$$

with

$$
f(x, t)=\frac{c_{1} t x^{2 m-7}}{m} \quad \text { and } \quad g(x, t)=\frac{c_{2} t x^{2 n-5}}{n},
$$

being $c_{1}$ and $c_{2}$ arbitrary constants. The choice of symmetries $\left\{\mathbf{X}_{1}, \mathbf{X}_{4}\right\},\left\{\mathbf{X}_{2}, \mathbf{X}_{4}\right\}$ and $\left\{\mathbf{X}_{3}, \mathbf{X}_{4}\right\}$ leads to the same result as $\left\{\mathbf{X}_{4}\right\}$.

Symmetry $\left\{\mathbf{X}_{3}\right\}$ : Following the prescription described, we have another class of $K(m, n)$ equations with variable coefficients

$$
f(u, x, t)=\frac{c_{1} t}{m} e^{(1-m) x / 2 t u}+\frac{2 u^{2-m}}{m(m-1)} \quad \text { and } \quad g(u, x, t)=\frac{c_{2} t}{n} e^{(1-n) x / 2 t u},
$$

with $c_{1}$ and $c_{2}$ as arbitrary constants.

Symmetries $\left\{\mathbf{X}_{1}, \mathbf{X}_{2}, \mathbf{X}_{3}, \mathbf{X}_{4}\right\}$ : Finally, replacing separately the coefficients of each generator $X_{i}$, $i=1,2,3,4$, into the system of determining Eqs. (2.6), we have obtained

$$
f(u, x, t)=\frac{c_{1} x t}{m} e^{(1-m) x / 2 t u} \quad \text { and } \quad g(u, x, t)=\frac{c_{2} t}{n} e^{(1-n) x / 2 t u},
$$

with arbitrary constants $c_{1}$ and $c_{2}$.

\section{General Remarks}

In this work we have used the known symmetry algebra of the classical KdV equation, $\ell_{K d V}$, to find and classify $K(m, n)$ equations with space- and time-dependent coefficients. This programme 
required an intensive use of computer algebra, and we have used the package SADE (Symmetry Analysis of Differential Equations) [11] for solving the determining equations. These equations with variable coefficients are particularly useful to understand the nonlinear mechanism that underlies processes described by the $K(m, n)$ equations. For this class of nonlinear KdV-type equations, a study of symmetry invariant solutions is in progress.

\section{Acknowledgments}

The authors thank A. E. Santana for a critical reading of the manuscript and useful comments. This work was supported by CAPES and FAPEMAT (Brazil).

\section{References}

[1] P. Rosenau and J. M. Hyman, Compactons: solitons with finite wavelength, Phys. Rev. Lett. 70 (1993) 564.

[2] P. Bracken, Symmetry properties of a generalized Korteweg-de Vries equation and some explicit solutions, Int. J. Math. Math. Sciences 13 (2005) 2159.

[3] P. Rosenau, Nonlinear dispersion and compact structures, Phys. Rev. Lett. 73 (1994) 1737.

[4] A. Pikovsky, P. Rosenau, Phase compactons, Physica D 218 (2006) 56.

[5] P. Rosenau, On a model equation of travelling and stationary compactons, Phys. Lett. A 356 (2006) 44.

[6] Y. Wang, L. Wang and W. Zhang, Application of the Adomian decomposition method to fully nonlinear Sine-Gordon equation, Int. J. Nonl. Science 2 (2006) 29.

[7] K. Singh and R. K. Gupta, On symmetries and invariant solutions of a coupled KdV system with variable coefficients, Int. J. Math. Math. Sciences 23 (2005) 3711.

[8] F. Güngör, V. I. Lahno and R. Z. Zhdanov, Symmetry classification of KdV-type nonlinear evolution equations, J. Math. Phys. 45 (2004) 2280.

[9] A. H. Bokhari, A. H. Kara and F. D. Zaman, On soliton and other exact solutions of the combined KdV and modified and generalized KdV equations, IL NUOVO CIMENTO 120 (2005) 393.

[10] E. M. Silva, T. M. Rocha-Filho and A. E. Santana, Lie symmetries of Fokker-Planck equations with logarithmic diffusion and drift terms, J. Phys: Conf. Series 40 (2006) 150.

[11] T. M. Rocha Filho and A. Figueiredo, http://ares.fis.unb.br/fismat/sade.html 